Pacific

Journal of

Mathematics

\title{
ON DIOPHANTINE MONOIDS AND THEIR CLASS
} GROUPS

Scott T. Chapman, Ulrich Krause, and Eberhard Oeljeklaus 


\title{
ON DIOPHANTINE MONOIDS AND THEIR CLASS GROUPS
}

\author{
Scott T. Chapman, Ulrich Krause, and Eberhard Oelueklaus
}

\begin{abstract}
A Diophantine monoid $S$ is a monoid which consists of the set of solutions in nonnegative integers to a system of linear Diophantine equations. Given a Diophantine monoid $\boldsymbol{S}$, we explore its algebraic properties in terms of its defining integer matrix $A$. If $d_{r}(S)$ and $d_{c}(S)$ denote respectively the minimal number of rows and minimal number of columns of a defining matrix $A$ for $S$, then we prove in Section 3 that $d_{r}(S)=\operatorname{rank} \mathrm{Cl}(S)$ and $d_{c}(S)=\operatorname{rank} \mathrm{Cl}(S)+\operatorname{rank} Q(S)$ where $\mathrm{Cl}(S)$ represents the divisor class group of $S$ and $Q(S)$ the quotient group of $S$. The proof relies on the characteristic properties of the so-called essential states of $S$, which are developed in Section 2. We close in Section 4 by offering a characterization of factorial Diophantine monoids and an algorithm which determines if a Diophantine monoid is halffactorial.
\end{abstract}

\section{Introduction.}

Because of their applications in commutative algebra, algebraic geometry, combinatorics, number theory, and computational algebra, the study of commutative cancellative monoids has recently increased in popularity (see for example [14]). Let $\mathbb{Z}$ and $\mathbb{N}$ represent the integers and the nonnegative integers respectively. For $1 \leq m, n$ in $\mathbb{N}$ and $A \in \mathbb{Z}^{m \times n}$ set

$$
M_{A}=\mathbb{N}^{n} \cap\left\{x \in \mathbb{Z}^{n} \mid A x=0\right\} .
$$

We will refer to $M_{A}$ as a Diophantine monoid and to $A$ as a matrix which determines $M_{A}$. Admitting the possibility that $m=0$, we set $M_{A}=\mathbb{N}^{n}$ if $m=0$. The special case of a Diophantine monoid $M_{A}$ for $A \in \mathbb{Z}^{1 \times n}$ (i.e., one single homogeneous linear Diophantine equation) has been studied in [2], where it was shown that the divisor class group of $M_{A}$ (denoted $\mathrm{Cl}\left(M_{A}\right)$ ) in this case must be cyclic [2, Theorem 1.3].

It is natural to consider the question of whether a Diophantine monoid $M_{A}$ given by a matrix $A \in \mathbb{Z}^{m \times n}$ can be described (up to isomorphy) by another matrix with less rows or less columns. For a Diophantine monoid $S$, let $d_{r}(S)$ and $d_{c}(S)$ denote respectively the minimal number of rows and minimal number of columns of a matrix $A$ with $S \simeq M_{A}$. One has that 
$d_{c}(S)=d_{r}(S)+\operatorname{rank} Q(S)$ where $Q(S)$ is the quotient group of $S$. In this paper, we prove for a monoid which is root-closed and finitely generated that $d_{r}(S)=\operatorname{rank} \mathrm{Cl}(S)$ and hence $d_{c}(S)=\operatorname{rank} \mathrm{Cl}(S)+\operatorname{rank} Q(S)$ (Theorem 3.8). Furthermore, this theorem also shows that for such an $S$ there exists an $A \in \mathbb{Z}^{m \times n}$ with $m=d_{r}(S), n=d_{c}(S)$ and $S \simeq M_{A}$.

More precisely, we divide our work into three additional sections. After a very brief review of Krull monoids, we focus our considerations in Section 2 on the so-called essential states of these monoids and provide in Lemma 2.3 and Proposition 2.4 their characteristic properties. Using these properties, we obtain the above mentioned result (Theorem 3.8) from our crucial Theorem 3.1 together with Lemma 3.5. Theorem 3.1 demonstrates that any Krull monoid with finitely many essential states must be isomorphic to a Diophantine monoid and describes the structure of the representing matrix as well as the structure of the class group. As a consequence, we obtain in Corollary 3.3 various equivalent characterizations of the above Krull monoids or, equivalently, Krull monoids with finitely generated divisor class group and finitely many prime divisors. For some results related to Corollary 3.3, the interested reader can consult [7, Theorem 5], [8, Lemma 3], [10, Proposition $2]$, and [13, Corollary 1]. We note that Lemma 3.5 yields a description of the class group of a Diophantine monoid $S$ purely in terms of linear algebra. In Section 4, we present some examples to illustrate the results of Sections 2 and 3 and also obtain in Proposition 4.1 a characterization of when $M_{A}$ is factorial (recall that $M_{A}$ is factorial if any nonzero element has a representation $\alpha_{1}+\cdots+\alpha_{k}$ by irreducible elements $\alpha_{i}$ which is unique up to ordering). We close by presenting an algorithm, which when teamed with known algorithms for computing the set of minimal nonnegative solutions to $M_{A}$, will compute the class group of $M_{A}$ and determine if $M_{A}$ is half-factorial (recall that $M_{A}$ is half-factorial if whenever $\alpha_{1}, \alpha_{2}, \ldots, \alpha_{k}$ and $\beta_{1}, \beta_{2}, \ldots, \beta_{l}$ are irreducible elements of $M_{A}$ with $\alpha_{1}+\cdots+\alpha_{k}=\beta_{1}+\cdots+\beta_{l}$, then $k=l$ ).

Although the literature concerning the algebraic structure of the monoids $M_{A}$ is not extensive, we take interest in this topic partly because of the rich mathematical history behind the study of Diophantine equations. It is easy to determine the solution set of a system of linear Diophantine equations over the integers, but this is not the case for determining the set of solutions over the nonnegative integers. While a modern treatment of the combinatorial aspects of this subject can be found in the works of Stanley (see [15] and [16] for example), attempts to determine the set of "irreducible solutions" of $M_{A}$ can be traced back almost 100 years to a paper of Elliott [4], where the author produces generating functions to determine these solutions. Another early attempt at producing this set of minimal solutions can be found in [5]. The development of modern algorithms connected with these solutions has become a popular topic of research in computational algebra (see [3] and [14]). 


\section{Essential states.}

Let $S$ be a commutative cancellative monoid (i.e., a subsemigroup of an abelian group written additively with $0 \in S$ ). Throughout this paper we assume that $S \neq\{0\}$. If $\{0\}$ is the only subgroup of $S$, then $S$ is called reduced. Let

$$
Q(S):=\{x-y \mid x, y \in S\}
$$

be the group generated by $S$. A homomorphism $\pi: Q(S) \rightarrow \mathbb{Z}$ is called a state of $S$ if $\pi(S) \subseteq \mathbb{N}$. The monoid $S$ is a Krull monoid if there exists, for some set $K$, a monomorphism

$$
\varphi: Q(S) \rightarrow \mathbb{Z}^{(K)} \quad(\text { direct sum })
$$

such that

$$
\varphi(S)=\mathbb{N}^{(K)} \cap \varphi(Q(S))=\mathbb{N}^{(K)} \cap Q(\varphi(S)) .
$$

As a consequence of our definition, a Krull monoid is always reduced.

For $j \in K$ let $p_{j}: \mathbb{Z}^{(K)} \rightarrow \mathbb{Z}$ be the surjection onto the $j$-th component, $p_{j}\left(\left(x_{k}\right)_{k \in K}\right)=x_{j}$, and

$$
\pi_{j}: Q(S) \rightarrow \mathbb{Z}, \quad \pi_{j}=p_{j} \circ \varphi .
$$

Then

$$
S=\left\{x \in Q(S) \mid \pi_{j}(x) \geq 0 \text { for all } j \in K\right\}
$$

where $\left(\pi_{j}\right)_{j \in K}$ is a family of states of $S$ with $\pi_{j}(x)=0$ for almost all $j \in K$ and any fixed $x \in Q(S)$. We may assume that $\pi_{j} \neq 0$ for all $j \in K$.

Example 2.1. For every $A \in \mathbb{Z}^{m \times n}$ the Diophantine monoid

$$
M_{A}=\left\{x \in \mathbb{N}^{n} \mid A x=0\right\} \subseteq \mathbb{Z}^{n}
$$

is a Krull monoid. With $K=\{1,2, \ldots, n\}$ and $\varphi: Q\left(M_{A}\right) \rightarrow \mathbb{Z}^{n}$ the canonical embedding, we have

$$
M_{A}=\left\{\left(x_{1}, \ldots, x_{n}\right) \in Q\left(M_{A}\right) \mid \pi_{j}(x)=x_{j} \geq 0 \text { for } j \in K\right\} .
$$

When a Krull monoid $S$ is given in the form $(*)$, it is natural to ask for minimal subsets $E \subseteq K$ with the property that

$$
S=\left\{x \in Q(S) \mid \pi_{i}(x) \geq 0 \text { for all } i \in E\right\} .
$$

Such subsets exist and can be described by the so-called essential states of $S$.

Definition 2.2. A nonzero state $\pi$ of $S$ is called essential, if for every $x, y \in$ $Q(S)$ with $\pi(x) \geq \pi(y)$ there exists $z \in Q(S)$ with

$$
\pi(z)=\pi(x), z-x \in S \text { and } z-y \in S .
$$

The essential states can be characterized as follows: 
Lemma 2.3. Let $S$ be a Krull monoid and $\left(\pi_{j}\right)_{j \in J}$ the family of all nonzero states of $S$. For every $i \in J$ the following statements are equivalent:

i) $\pi_{i}$ is essential.

ii) For every $j \in J$ with $\pi_{j} \notin \mathbb{Q} \pi_{i}$ there exists $x \in S$ such that $\pi_{i}(x)=0$ and $\pi_{j}(x)>0$.

iii) $S \cap \operatorname{Ker} \pi_{i}$ is a maximal element in the set $\left\{S \cap \operatorname{Ker} \pi_{j} \mid j \in J\right\}$ with respect to set inclusion.

iv) If $j \in J$ and $\left(S \cap \operatorname{Ker} \pi_{i}\right) \subseteq\left(S \cap \operatorname{Ker} \pi_{j}\right)$ then $\pi_{j}=\alpha \pi_{i}$ for some $\alpha \in \mathbb{Q}, \alpha>0$.

In particular, the family of essential states of $S$ is not empty.

Proof. Note that for any finite subset $I \subseteq J$, there exists $x \in S$ with $\pi_{i}(x)>$ 0 for all $i \in I$.

Statements ii) and iv) are obviously equivalent. We begin by showing that i) $\Rightarrow$ iii). Assume that i) holds and that, for some $j \in J$,

$$
\left(S \cap \operatorname{Ker} \pi_{i}\right) \varsubsetneqq\left(S \cap \operatorname{Ker} \pi_{j}\right) .
$$

Choose $y \in\left(S \cap \operatorname{Ker} \pi_{j}\right) \backslash \operatorname{Ker} \pi_{i}$ and $x \in S$ with $\pi_{j}(x)>0$. Then

$$
u:=\pi_{i}(y) x-\pi_{i}(x) y \in \operatorname{Ker} \pi_{i} .
$$

Condition i) yields an element $z \in Q(S)$ with

$$
\begin{gathered}
\pi_{i}(z)=\pi_{i}\left(\pi_{i}(y) x\right)=\pi_{i}\left(\pi_{i}(x) y\right), \\
z-\pi_{i}(y) x \in S \cap \text { Ker } \pi_{i} \subseteq \operatorname{Ker} \pi_{j}
\end{gathered}
$$

and

$$
z-\pi_{i}(x) y \in S \cap \operatorname{Ker} \pi_{i} \subseteq \operatorname{Ker} \pi_{j} .
$$

In particular, $\pi_{j}\left(z-\pi_{i}(y) x\right)=\pi_{j}\left(z-\pi_{i}(x) y\right)=0$. But this gives

$$
0=\pi_{j}\left(z-\pi_{i}(x) y\right)=\pi_{j}(z)-\pi_{i}(x) \pi_{j}(y)=\pi_{j}(z),
$$

and the contradiction

$$
0=\pi_{j}\left(z-\pi_{i}(y) x\right)=\pi_{j}(z)-\pi_{i}(y) \pi_{j}(x)=-\pi_{i}(y) \cdot \pi_{j}(x)<0 .
$$

For the rest of the proof, let $K \subseteq J$ be a subset of $J$ with the property that

$$
S=\left\{x \in Q(S) \mid \pi_{j}(x) \geq 0 \text { for all } j \in K\right\}
$$

where $\pi_{j}(x)=0$ for almost all $j \in K$ and any fixed $x \in Q(S)$. The representation $(*)$ shows that such subsets $K$ exist.

For iii) $\Rightarrow$ iv), we fix $i \in J$ and assume that iii) is true for $\pi_{i}$. Moreover we fix $j \in J$ and assume that $S \cap \operatorname{Ker} \pi_{i} \subseteq S \cap \operatorname{Ker} \pi_{j}$. Define $L:=K \cup\{i, j\}$. 
From iii) we know that

$$
\begin{aligned}
L_{0}: & =\left\{k \in L \mid\left(S \cap \operatorname{Ker} \pi_{i}\right) \subseteq \operatorname{Ker} \pi_{k}\right\} \\
& =\left\{k \in L \mid S \cap \operatorname{Ker} \pi_{i}=S \cap \operatorname{Ker} \pi_{k}\right\} .
\end{aligned}
$$

We choose some $v \in S$ with $\pi_{i}(v)>0$. Then $\pi_{j}(v)>0$ by our assumption and $\alpha=\frac{\pi_{j}(v)}{\pi_{i}(v)}>0$. Let $w \in S$ be arbitrary. If $\pi_{i}(w)=0$, then $\pi_{j}(w)=0$ and $\pi_{i}(w)=\alpha \pi_{j}(w)$. Assume that $\pi_{i}(w)>0$. Then

$$
i \in L_{0}^{\prime}:=\left\{k \in L_{0} \mid \pi_{k}(w) \neq 0\right\} .
$$

Since $L_{0}^{\prime}$ is finite, we can define $m=\prod_{k \in L_{0}^{\prime}} \pi_{k}(w)$ and

$$
\lambda=\max \left\{r \in \mathbb{N} \mid r \geq 1, \pi_{k}(m v-r w) \geq 0 \text { for all } k \in L_{0}\right\} .
$$

For some $k_{1} \in L_{0}^{\prime}$ the equality

$$
\pi_{k_{1}}(m v-\lambda w)=0
$$

holds. For every $k \in L_{2}:=\left\{k \in L \backslash L_{0} \mid \pi_{k}(m v-\lambda w) \neq 0\right\}$ we choose $x_{k} \in S \cap \operatorname{Ker} \pi_{i}$ with

$$
\pi_{k}\left(x_{k}\right)>\max \left\{0, \pi_{k}(\lambda w-m v)\right\} .
$$

Since $L_{2}$ is finite (and possibly empty), the element $x:=\sum_{\mu \in L_{2}} x_{\mu}$ is welldefined and $x \in S \cap \operatorname{Ker} \pi_{i} \subseteq$ Ker $\pi_{j}$.

In the next step, we show that $u=x+m v-\lambda w \in S$ (i.e., that $\pi_{k}(u) \geq 0$ for all $k \in L)$. We have already seen that for $k \in L_{0}$ we have

$$
\pi_{k}(u)=\pi_{k}(x)+\pi_{k}(m v-\lambda w) \geq \pi_{k}(m v-\lambda w) \geq 0 .
$$

For $k \in L_{2}$ we get

$$
\pi_{k}(u)=\pi_{k}(x)+\pi_{k}(m v-\lambda w) \geq \pi_{k}\left(x_{k}\right)+\pi_{k}(m v-\lambda w) \geq 0,
$$

and finally for $k \in L \backslash\left(L_{0} \cup L_{2}\right)=\left(L \backslash L_{0}\right) \backslash L_{2}$ we have that

$$
\pi_{k}(u)=\pi_{k}(x) \geq 0 .
$$

Therefore $u \in S$, and it follows for the above chosen $k_{1} \in L_{0}^{\prime}$ with $\pi_{k_{1}}(m v-$ $\lambda w)=0$ that

$$
\pi_{k_{1}}(u)=\pi_{k_{1}}(x)+\pi_{k_{1}}(m v-\lambda w)=\pi_{k_{1}}(x)=0,
$$

since $x \in S \cap \operatorname{Ker} \pi_{i}=S \cap \operatorname{Ker} \pi_{k_{1}}$. In particular, $u \in S \cap \operatorname{Ker} \pi_{i} \subseteq \operatorname{Ker} \pi_{j}$. This gives

$$
\pi_{j}(u)=0=\pi_{i}(u)=\pi_{i}(m v-\lambda w)=\pi_{j}(m v-\lambda w) .
$$

Hence

$$
\lambda \pi_{j}(w)=\pi_{j}(m v), \lambda \pi_{i}(w)=\pi_{i}(m v)
$$

and

$$
\pi_{j}(w)=\frac{1}{\lambda} \pi_{j}(m v)=\frac{\pi_{j}(m v)}{\pi_{i}(m v)} \pi_{i}(w)=\frac{\pi_{j}(v)}{\pi_{i}(v)} \pi_{i}(w)=\alpha \pi_{i}(w) .
$$


Since $w \in S$ was chosen arbitrarily, it follows that $\pi_{j}=\alpha \pi_{i}$.

For iv) $\Rightarrow \mathrm{i}$ ), assume that $x, y \in Q(S)$ are given with $\pi_{i}(x) \geq \pi_{i}(y)$. As above, we define

$$
L:=K \cup\{i, j\}, L_{0}:=\left\{k \in L \mid\left(S \cap \operatorname{Ker} \pi_{i}\right) \subseteq \operatorname{Ker} \pi_{k}\right\}, L_{1}:=L \backslash L_{0},
$$

and $L_{2}:=\left\{k \in L_{1} \mid \pi_{k}(x-y) \neq 0\right\}$. Note that $L_{2}$ is a finite set. By iv) we know that

$$
\pi_{k}=\alpha_{k} \pi_{i}, \quad \text { for all } k \in L_{0} \text { where } 0<\alpha_{k} \in \mathbb{Q} .
$$

For every $k \in L_{1}$ there exists $x_{k} \in S \cap \operatorname{Ker} \pi_{i}$ with

$$
\pi_{k}(x-y)+\pi_{k}\left(x_{k}\right) \geq 0 .
$$

Defining $z=x+\sum_{\mu \in L_{2}} x_{\mu}$, we get

$$
z-x=\sum_{\mu \in L_{2}} x_{\mu} \in S .
$$

We prove that $z-y \in S$ by showing that $\pi_{k}(z-y) \geq 0$ for all $k \in L$.

If $k \in L_{2}$, then

$$
\begin{aligned}
\pi_{k}(z-y) & =\pi_{k}\left(x-y+\sum_{\mu \in L_{2}} x_{\mu}\right)=\pi_{k}(x-y)+\sum_{\mu \in L_{2}} \pi_{k}\left(x_{\mu}\right) \\
& =\pi_{k}(x-y)+\pi_{k}\left(x_{k}\right)+\sum_{\mu \in L_{2} \backslash\{k\}} \pi_{k}\left(x_{\mu}\right) \geq 0 .
\end{aligned}
$$

For $k \in L_{0}$ we get

$$
\pi_{k}(z-y)=\pi_{k}(x-y)=\alpha_{k} \pi_{i}(x-y) \geq 0 .
$$

Finally, for $k \in L_{1} \backslash L_{2}$ we have

$$
\pi_{k}(z-y)=\pi_{k}\left(\sum_{\mu \in L_{2}} x_{\mu}\right) \geq 0 .
$$

Again, let the Krull monoid $S$ be given in the form (*). Thus,

$$
S=\left\{x \in Q(S) \mid \pi_{j}(x) \geq 0 \text { for all } j \in K\right\}
$$

where $\left(\pi_{j}\right)_{j \in K}$ is a family of nonzero states of $S$ such that $\pi_{j}(x)=0$ for almost all $j \in K$ and any fixed $x \in Q(S)$.

Proposition 2.4. For every $\emptyset \neq I \subseteq K$ the following statements are equivalent:

i) $S=\left\{x \in Q(S) \mid \pi_{i}(x) \geq 0\right.$ for all $\left.i \in I\right\}$. 
ii) If $\pi$ is an essential state of $S$, then there exists $i \in I$ and $\alpha \in \mathbb{Q}, \alpha>0$, such that

$$
\pi=\alpha \pi_{i} .
$$

Proof. i) $\Longrightarrow$ ii) Let $\pi$ be an essential state of $S$. It suffices to show that $\pi \in \mathbb{Q} \pi_{i}$ for some $i \in I$. Let $i_{0} \in I$ and $v, w \in S$ with $\pi(v)>0, \pi_{i_{0}}(w)>0$ and define $u:=v+w$. Then $\pi(u)>0$ and

$$
i_{0} \in I_{1}:=\left\{i \in I \mid \pi_{i}(u)>0\right\}, \quad\left|I_{1}\right|<\infty .
$$

We claim that $\pi \in \mathbb{Q} \pi_{i}$ for some $i \in I_{1}$. Assume the contrary. Then $\pi_{i} \notin \mathbb{Q} \pi$ and we apply Lemma 2.3 ii) to get for every $i \in I_{1}$ an element $x_{i} \in S$ with $\pi\left(x_{i}\right)=0$ and $\pi_{i}\left(x_{i}\right)>0$. With $x:=\sum_{i \in I_{1}} x_{i} \in S$ it follows that $\pi(x)=0$ and for every $i \in I_{1}$

$$
\pi_{i}(x)=\pi_{i}\left(x_{i}\right)+\sum_{j \in I_{1} \backslash\{i\}} \pi_{i}\left(x_{j}\right)>0 .
$$

Let $i_{1} \in I_{1}$ with

$$
\frac{\pi_{i_{1}}(x)}{\pi_{i_{1}}(u)}=\min \left\{\frac{\pi_{i}(x)}{\pi_{i}(u)} \mid i \in I_{1}\right\}
$$

and $z:=\pi_{i_{1}}(u) x-\pi_{i_{1}}(x) u$. From i) we get $z \in S$, since

$$
\pi_{i}(z)=\pi_{i_{1}}(u) \pi_{i}(x)-\pi_{i_{1}}(x) \pi_{i}(u) \geq 0 \text { for } i \in I_{1},
$$

and

$$
\pi_{i}(z)=\pi_{i_{1}}(u) \pi_{i}(x) \geq 0 \text { for } i \in I \backslash I_{1} .
$$

But $\pi(z)=-\pi_{i_{1}}(x) \pi(u)<0$, a contradiction to the fact that $\pi(S) \subseteq \mathbb{N}$.

ii) $\Longrightarrow$ i) Let

$$
N:=\left\{w \in Q(S) \mid \pi_{i}(w) \geq 0 \text { for all } i \in I\right\} .
$$

For $y \in N$ we consider the finite set $K(y):=\left\{j \in K \mid \pi_{j}(y)<0\right\}$ and prove that $|K(y)|=0$ (i.e., that $A:=\{y \in N|| K(y) \mid \geq 1\}=\varnothing$ ). Assume the contrary and choose $y \in A$ such that $|K(y)|$ is minimal. Let $j_{0} \in K$ such that $\pi_{j_{0}}$ is an essential state of $S$. From ii) we know that $j_{0} \notin K(y)$. For every $j \in\left\{j_{0}\right\} \cup K(y)$ there exists $x_{j} \in S$ with $\pi_{j}\left(x_{j}\right)>0$. Thus $x:=\sum_{j \in\left\{j_{0}\right\} \cup K(y)} x_{j} \in S$ and $\pi_{j}(x)>0$ for every $j \in\left\{j_{0}\right\} \cup K(y)$. Let $j_{1} \in K(y)$ such that

$$
0>\frac{\pi_{j_{1}}(y)}{\pi_{j_{1}}(x)}=\max \left\{\frac{\pi_{j}(y)}{\pi_{j}(x)} \mid j \in K(y)\right\} .
$$

We define $z:=\pi_{j_{1}}(x) y-\pi_{j_{1}}(y) x$. Since $\pi_{j_{1}}(y)<0$ it follows for every $j \in K \backslash K(y)$ that

$$
\pi_{j}(z)=\pi_{j_{1}}(x) \pi_{j}(y)-\pi_{j_{1}}(y) \pi_{j}(x) \geq-\pi_{j_{1}}(y) \pi_{j}(x) \geq 0 .
$$


In particular, $z \in N$ since $I \subseteq K \backslash K(y)$, and $K(z) \subseteq K(y)$. Moreover $|K(z)|<|K(y)|$ because $j_{1} \notin K(z)$. From the minimality of $|K(y)|$ we conclude $|K(z)|=0$ (i.e., $z \in S$ ). Since

$$
\pi_{j_{0}}(z)=\pi_{j_{1}}(x) \pi_{j_{0}}(y)-\pi_{j_{1}}(y) \pi_{j_{0}}(x) \geq-\pi_{j_{1}}(y) \pi_{j_{0}}(x)>0
$$

it follows that

$$
z \in\left(S \cap \operatorname{Ker} \pi_{j_{1}}\right) \nsubseteq \subseteq\left(S \cap \operatorname{Ker} \pi_{j_{0}}\right) .
$$

But, by Lemma 2.3 iii), there has to be an essential state $\pi$ of $S$ with

$$
\left(S \cap \operatorname{Ker} \pi_{j_{1}}\right) \subseteq(S \cap \operatorname{Ker} \pi) .
$$

Since $\pi_{j_{0}}$ was already arbitrarily chosen, this contradiction finishes the proof.

We call a state $\pi$ of $S$ a normal state, if $\pi(Q(S))=\mathbb{Z}$. For every nonzero state $\pi: Q(S) \rightarrow \mathbb{Z}$, the image $\pi(Q(S))$ is a nonzero ideal $d \mathbb{Z}$ of $\mathbb{Z}$ where $d \in \mathbb{N}, d \geq 1$, and $\pi_{\text {nor }}:=\frac{1}{d} \pi$ is normal. Let $K_{N}$ denote the set of all normal states of a given Krull monoid $S$. From Proposition 2.4 we obtain the following.

Corollary 2.5. There is a unique minimal subset $I(S) \subseteq K_{N}$ such that

$$
S=\{x \in Q(S) \mid \pi(x) \geq 0 \text { for all } \pi \in I(S)\} .
$$

The set $I(S)$ consists exactly of the essential normal states of $S$. Moreover $\pi(x)=0$ for almost all $\pi \in I(S)$ and any fixed $x \in Q(S)$.

Proof. Let $L$ be a set of nonzero states of $S$ such

$$
S=\{x \in Q(S) \mid \pi(x) \geq 0 \text { for all } \pi \in L\}
$$

and $\pi(x)=0$ for almost all $\pi \in L$ and any fixed $x \in Q(S)$. Define $\widetilde{L}:=$ $\left\{\pi_{\text {nor }} \mid \pi \in L\right\}$. Obviously $S=\left\{x \in Q(S) \mid \pi_{\text {nor }}(x) \geq 0\right.$ for all $\left.\pi \in L\right\}$, and the set $I(S)$ of normal essential states of $S$ is a subset of $\widetilde{L}$ by Proposition 2.4. In particular $\pi(x)=0$ for almost all $\pi \in I(S)$ and any fixed $x \in Q(S)$. Now we may again apply Proposition 2.4 and conclude that $S=\{x \in$ $Q(S) \mid \pi(x) \geq 0$ for all $\pi \in I(S)\}$.

We consider the form

$$
S=\{x \in Q(S) \mid \pi(x) \geq 0 \text { for all } \pi \in I(S)\}
$$

as the (uniquely determined) normal representation of the Krull monoid $S$. It gives rise to a divisor theory

$$
\begin{aligned}
& \varphi: Q(S) \rightarrow \mathbb{Z}^{(I(S))}, \quad \text { (direct sum) } \\
& \varphi(x):=(\pi(x))_{\pi \in I(S)},
\end{aligned}
$$


with $\operatorname{Ker} \varphi=S \cap(-S)=\{0\}$, which maps $Q(S)$ onto a free subgroup of $\mathbb{Z}^{(I(S))}$ such that

$$
\varphi(S)=\mathbb{N}^{(I(S))} \cap \varphi(Q(S))=\mathbb{N}^{(I(S))} \cap Q(\varphi(S)) .
$$

The quotient group

$$
\mathrm{Cl}(S):=\mathbb{Z}^{(I(S))} / \varphi(Q(S))
$$

is the divisor class group of $S$. It is a direct consequence of the construction that isomorphic Krull monoids have isomorphic divisor class groups.

Now suppose that $A \in \mathbb{Z}^{m \times n}$ and $S$ is isomorphic to the Diophantine monoid

$$
M_{A}=\left\{x \in \mathbb{N}^{n} \mid A x=0\right\} .
$$

For $1 \leq i \leq n$ let $p_{i}: \mathbb{Z}^{n} \rightarrow \mathbb{Z}$, with $p_{i}\left(x_{1}, \ldots, x_{n}\right)=x_{i}$, denote the natural surjection. The restriction maps $\pi_{i}=p_{i} \mid Q\left(M_{A}\right)$ from $Q\left(M_{A}\right)$ into $\mathbb{Z}$ are states of $M_{A}$. We call them the canonical projections of $Q\left(M_{A}\right)$. Note that a relation $\pi_{i}=\pi_{j}$ does not imply $i=j$. Moreover, for $1 \leq i \leq n$ we define $c_{i}=\operatorname{gcd}\left(x_{i} \mid x=\left(x_{1}, \ldots, x_{n}\right) \in M_{A}\right) \in \mathbb{N}$ where $c_{i}=0$ if and only if $x_{i}=\pi_{i}(x)=0$ for all $x \in M_{A}$. We call the product $w\left(M_{A}\right)=\prod_{i=1}^{n} c_{i}$ the weight of the monoid $M_{A}$. If $n \geq 2$ and $c_{i}=0$, then $M_{A}$ is canonically isomorphic to the monoid $M_{\widetilde{A}} \subseteq \mathbb{N}^{n-1}$, where $\widetilde{A} \in \mathbb{Z}^{m \times(n-1)}$ is given by canceling the $i$-th column of $A$. Therefore it suffices to study the situation $w\left(M_{A}\right) \neq 0$. All canonical projections $\pi_{i}, 1 \leq i \leq n$, are normal if and only if $w\left(M_{A}\right)=1$. In this case, every normal essential state of $M_{A}$ is a canonical projection (as follows immediately from Corollary 2.5), hence there are at most $n$ essential states of $M_{A}$. In general, not all normal canonical projections of $Q\left(M_{A}\right)$ are essential states of $M_{A}$. Let

$$
I\left(M_{A}\right):=\left\{i \mid 1 \leq i \leq n, \pi_{i} \text { is an essential state of } M_{A}\right\}
$$

and note that the following statements are equivalent:

i) $w\left(M_{A}\right) \neq 0$,

ii) there exist elements $x=\left(x_{1}, \ldots, x_{n}\right) \in M_{A}$ with $x_{i}>0$ for $1 \leq i \leq n$.

Lemma 2.6. Let $M_{A}$ be a Diophantine monoid with $A \in \mathbb{Z}^{m \times n}$ and $w\left(M_{A}\right)$ $\neq 0$. The following statements are true:

i) $Q\left(M_{A}\right)=\left\{x \in \mathbb{Z}^{n} \mid A x=0\right\}$, and there exists a linearly independent system of vectors $v_{1}, v_{2}, \ldots v_{n-r} \in M_{A}$, where $r=\operatorname{rank} A$.

ii) Let $c_{i}$ be defined as above and $D:=\operatorname{diag}\left(c_{1}, \ldots, c_{n}\right) \in \mathbb{Z}^{n \times n}$. The map $M_{A D} \rightarrow M_{A}$, defined by $y \mapsto D y$, is an isomorphism of monoids and $w\left(M_{A D}\right)=1$.

iii) There are at most $n$ normal essential states of $M_{A}$. 
Proof. i): The assumption $w\left(M_{A}\right) \neq 0$ yields an element $x=\left(x_{1}, \ldots, x_{n}\right) \in$ $M_{A}$ with $x_{i}>0$ for $1 \leq i \leq n$. Obviously $Q\left(M_{A}\right) \subseteq G=\left\{u \in \mathbb{Z}^{n} \mid A u=0\right\}$. If $y \in G$ then $z=k x-y \in \mathbb{N}^{n} \cap G=M_{A}$ for $k \in \mathbb{N}$ sufficiently big, hence $y=k x-z \in Q\left(M_{A}\right)$ and $Q\left(M_{A}\right)=G$. Let $r=\operatorname{rank} A$ and $w_{1}, \ldots, w_{n-r}$ be a basis of the $\mathbb{Q}$-vector space $W=\left\{z \in \mathbb{Q}^{n} \mid A z=0\right\}$. We may assume that $w_{j} \in \mathbb{Z}^{n}$ for $1 \leq j \leq n-r$. Let $m_{0} \in \mathbb{N}$ with $m_{0} x+w_{j} \in M_{A}, 1 \leq j \leq n-r$. Since the family $\left(k x+w_{j} \mid 1 \leq j \leq n-r\right)$ is linearly independent for all but at most one $k \in \mathbb{N}$, we are done.

We leave the verification of statements ii) and iii) to the reader.

\section{The representation of Krull monoids by matrices.}

In this section, we show that any Krull monoid $S$ with a finite number $e$ of essential states must be isomorphic to a Diophantine monoid. We describe the structure of the corresponding matrix and show in principle its computation, as well as the computation of the divisor class group of $S$. For the class group $\mathrm{Cl}(S)$ defined in the previous section, we have the short exact sequence

$$
0 \longrightarrow Q(S) \stackrel{\varphi}{\longrightarrow} \mathbb{Z}^{(I)} \stackrel{\rho}{\longrightarrow} \mathrm{Cl}(S) \longrightarrow 0
$$

where $I=I(S)$ indexes the set of all normalized essential states of $S$ and where $\rho$ denotes the canonical epimorphism. To obtain the desired description of $S$ by a matrix, we will use a particular basis for the $\mathbb{Z}$-module $\mathbb{Z}^{(I)}$. For an arbitrary $\mathbb{Z}$-module $M$, let $\operatorname{rank} M$ denote the minimal number of generators of $M$ and free rank $M$ the maximal length of a free family in $M$. Of course, if $M$ is a free module, then the rank and the free rank of $M$ coincide and are equal to the cardinality of a $\mathbb{Z}$-basis of $M$ (since $M=\{0\}$ is generated by the empty set, one has rank $\{0\}=$ free rank $\{0\}=0)$.

Theorem 3.1. For a (reduced) Krull monoid $S$, the number e of essential states is finite if and only if the rank $r$ of $Q(S)$, the free rank $h$ of $\mathrm{Cl}(S)$, and the rank $k$ of the torsion group of $\mathrm{Cl}(S)$ are all finite. In this case, the following statements apply.

i) There exist natural numbers $\alpha_{1}, \ldots, \alpha_{k} \geq 2$ with $\alpha_{i+1} \mid \alpha_{i}$ for $1 \leq i \leq$ $k-1$ such that

$$
\mathrm{Cl}(S) \simeq \mathbb{Z}_{\alpha_{1}} \oplus \cdots \oplus \mathbb{Z}_{\alpha_{k}} \oplus \mathbb{Z}^{h}
$$

and $h+r=e$.

ii) $S$ is isomorphic to a Diophantine monoid $M_{A}$ with $A \in \mathbb{Z}^{m \times n}$ for $m=k+h$ and $n=k+e\left(\right.$ if $h=k=0$, then $m=0$ and $\left.M_{A}=\mathbb{N}^{e}\right)$.

iii) The matrix A has a block structure 


$A=$\begin{tabular}{|l|l|}
\hline$A_{11}$ & $A_{12}$ \\
\hline$A_{21}$ & 0 \\
\hline
\end{tabular}

where $A_{11} \in \mathbb{N}^{k \times e}$ with entries of the $i$-th row in $\left\{0,1, \ldots, \alpha_{i}-1\right\}$, $A_{12}=-\operatorname{diag}\left(\alpha_{1}, \ldots, \alpha_{k}\right) \in \mathbb{N}^{k \times k}$ and $A_{21} \in \mathbb{N}^{h \times e}$ contains in each row at least two strictly positive and two strictly negative entries.

Proof. $M=\varphi(Q(S))$ is a submodule of the free module $\mathbb{Z}^{(I)}$ and therefore free. If $e=|I|$ is finite then $\operatorname{rank} M$ is finite and, hence, $r$ is finite. By its definition as the quotient of $\mathbb{Z}^{(I)}$ and $M$, the class group is finitely generated and $h$ and $k$ are finite. Conversely, if $r, h$ and $k$ are finite then

$$
k+h=\operatorname{rank} \mathrm{Cl}(S) \geq \operatorname{rank} \mathbb{Z}^{(I)}-\operatorname{rank} \varphi(Q(S))
$$

and hence $e \leq k+h+r$ is finite.

Step I. In a first step, we analyze certain submodules of $\mathbb{Z}^{(I)}$ for an arbitrary index set $I$. Let $\left(b_{i}\right)_{i \in I}$ be a basis of the free module $\mathbb{Z}^{(I)}$. For a given set $\left\{\alpha_{j} \mid j \in J\right\} \subseteq \mathbb{N} \backslash\{0\}, \emptyset \neq J \subseteq I$, let $M$ be the free submodule with basis $\left(\alpha_{j} b_{j}\right)_{j \in J}$. Consider the epimorphism $\psi: \mathbb{Z}^{(I)} \longrightarrow \bigoplus_{i \in J^{\prime}} \mathbb{Z}_{\alpha_{i}} \bigoplus \mathbb{Z}^{(I \backslash J)}$ for $J^{\prime}=\left\{j \in J \mid \alpha_{j} \neq 1\right\}$ defined by

$$
\psi\left(\sum_{i \in I} r_{i} b_{i}\right)=\sum_{i \in J^{\prime}}\left(r_{i} \bmod \alpha_{i}\right) b_{i}+\sum_{i \in I \backslash J} r_{i} b_{i} .
$$

Obviously, $\operatorname{Ker} \psi=M$ and, hence,

$$
\mathbb{Z}^{(I)} / M \simeq \bigoplus_{i \in J^{\prime}} \mathbb{Z}_{\alpha_{i}} \bigoplus \mathbb{Z}^{(I \backslash J)}
$$

To describe $M$ by a matrix, let $\left(u_{i}\right)_{i \in I}$ be the standard basis of $\mathbb{Z}^{(I)}$ and $u_{j}=\sum_{i \in I} c_{i j} b_{i}, c_{i j} \in \mathbb{Z}$ for $i, j \in I$. Define a block matrix

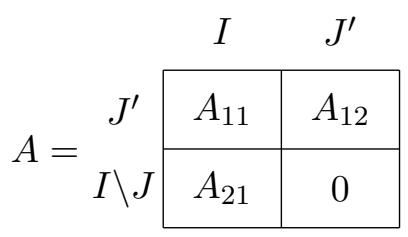

where the blocks in the above diagram are given as follows: 
$A_{11}: \quad$ For $i \in J^{\prime}, j \in I$, let $a_{i j} \in\left\{0,1, \ldots, \alpha_{i}-1\right\}$ be the residue of $c_{i j} \bmod \alpha_{i}$.

$A_{12}: \quad$ For $i, j \in J^{\prime}$, let $a_{i j}=-\alpha_{i} \delta_{i j}$.

$A_{21}$ : For $i \in I \backslash J, j \in I$, let $a_{i j}=c_{i j}$.

Note that $A_{21}$ cannot have a zero-row. Namely, $c_{i_{0} j}=0$ for $i_{0} \in I \backslash J$ and all $j \in I$ would imply $u_{j}=\sum_{i \neq i_{0}} c_{i j} b_{i}$ for all $j \in I$, which contradicts the freeness of $\left(b_{i}\right)_{i \in I}$.

For $N_{A}:=\left\{(x, y) \in \mathbb{Z}^{(I)} \times \mathbb{Z}^{\left(J^{\prime}\right)} \mid A\left[\begin{array}{l}x \\ y\end{array}\right]=0\right\}$ and $\pi: \mathbb{Z}^{(I)} \times \mathbb{Z}^{\left(J^{\prime}\right)} \longrightarrow$ $\mathbb{Z}^{(I)}$, defined by $\pi(x, y)=x$, we shall show that $\pi: N_{A} \longrightarrow M$ is an isomorphism of $\mathbb{Z}$-modules.

For $x \in \mathbb{Z}^{(I)}$ we have that

$$
x=\sum_{j} x_{j} u_{j}=\sum_{j} x_{j} \sum_{i} c_{i j} b_{i}=\sum_{i}\left(\sum_{j} c_{i j} x_{j}\right) b_{i} .
$$

Therefore, $x \in M$ if and only if

$$
\alpha_{i} \mid \sum_{j} c_{i j} x_{j} \text { for } i \in J^{\prime} \text { and } \sum_{j} c_{i j} x_{j}=0 \text { for } i \in I \backslash J,
$$

which is equivalent to

(4) $\sum_{j \in I} a_{i j} x_{j}=\alpha_{i} y_{i}$ with $y_{i} \in \mathbb{Z}$ for $i \in J^{\prime}$ and $\sum_{j \in I} a_{i j} x_{j}=0$ for $i \in I \backslash J$.

According to the definition of $A$, this means that $x \in M$ if and only if $(x, y) \in N_{A}$ for some $y \in \mathbb{Z}^{\left(J^{\prime}\right)}$. Thus, $\pi: N_{A} \longrightarrow M$ is a well-defined epimorphism. $\pi$ is injective because by (4) $y=\left(y_{i}\right)_{i \in J^{\prime}}$ is uniquely determined by $x=\left(x_{j}\right)_{j \in I}$. This shows that $\pi: N_{A} \longrightarrow M$ is an isomorphism.

Step II. Consider now a (reduced) Krull monoid $S$ for which $I=I(S)=$ $\{1, \ldots, e\}$ is finite and let $M=\varphi(Q(S)) \subseteq \mathbb{Z}^{(I)}$. Since $\mathbb{Z}^{(I)}$ is finitely generated, by the elementary divisor theorem (see [9]) there exist a basis $\left(b_{i}\right)_{i \in I}$ of $\mathbb{Z}^{(I)}$ and numbers $\alpha_{j} \in \mathbb{N} \backslash\{0\}$ for $1 \leq j \leq r \leq e$ with $\alpha_{j} \neq 1$ for $1 \leq j \leq k \leq r$ and $\alpha_{j+1} \mid \alpha_{j}$ for $1 \leq j \leq k-1$ such that $\left(\alpha_{j} b_{j}\right)_{1 \leq j \leq r}$ is a basis of $M(k=0$ admitted). Obviously, $r=\operatorname{rank} \varphi(Q(S))=\operatorname{rank} Q(S)$. Setting $J=\{1, \ldots, r\}$ and $J^{\prime}=\{1, \ldots, k\}$, from (2) in Step I, we obtain for $M=\varphi(Q(S))$ that $\mathrm{Cl}(S)=\mathbb{Z}^{(I)} / M \simeq \bigoplus_{i=1}^{k} \mathbb{Z}_{\alpha_{i}} \bigoplus \mathbb{Z}^{I \backslash J}$.

It follows that $k$ is the rank of the torsion group of $\mathrm{Cl}(S)$ and that $e-r=$ $|I \backslash J|$ is the free rank of $\mathrm{Cl}(S)$. This proves Part i) of Theorem 3.1.

Further, for the matrix $A$ defined by (3) in Step I, we have that $\pi: N_{A} \longrightarrow$ $M$ is an isomorphism. Hence the equations in (4) show that $\pi(x, y) \in$ $\mathbb{N}^{I}$ implies $(x, y) \in \mathbb{N}^{I} \times \mathbb{N}^{J^{\prime}}$. Since $S$ is a Krull monoid it follows that 
$\varphi(S)=\mathbb{N}^{I} \cap \varphi(Q(S))=\mathbb{N}^{I} \cap M$. Combining these facts, we obtain for $M_{A}:=\left(\mathbb{N}^{I} \times \mathbb{N}^{J^{\prime}}\right) \cap N_{A}$ that $S \stackrel{\varphi}{\longrightarrow} \mathbb{N}^{I} \cap M \stackrel{\pi^{-1}}{\longrightarrow} M_{A}$. Since $\varphi$ and $\pi^{-1}$ are monoid isomorphisms, we have that $S$ is isomorphic to the Diophantine monoid $M_{A}$.

Also, by (3) of Step I, the matrix $A$ is a block matrix where $A_{11} \in \mathbb{Z}^{k \times e}$ with entries $a_{i j} \in\left\{0,1, \ldots, \alpha_{i}-1\right\}, A_{12}=-\operatorname{diag}\left(\alpha_{1}, \ldots, \alpha_{k}\right) \in \mathbb{N}^{k \times k}$ and $A_{21} \in \mathbb{Z}^{h \times e}$. In particular, $A \in \mathbb{Z}^{m \times n}$ with $m=k+h, n=k+e$. This proves Part ii) and the first part of iii) of Theorem 3.1.

It remains to show the statement about $A_{21}$ in iii). Pick $i \in I \backslash J$ and let $I_{+}=\left\{j \in I \mid a_{i j}>0\right\}$ and $I_{-}=\left\{k \in I \mid a_{i k}<0\right\}$. Since by Step I the submatrix $A_{21}$ cannot have a zero-row, we must have that $I_{+}$or $I_{-}$is nonempty. We shall show in fact that both $I_{+}$and $I_{-}$must be nonempty. Suppose that $j_{1} \in I_{+}$. Since $J \neq \emptyset$ and $i \in I \backslash J$ imply $|I| \geq 2$, there exists $j_{2} \in I, j_{2} \neq j_{1}$. Let $\left(\pi_{j}\right)_{j \in I}$ be the set of normalized essential states of $S$. By the isomorphism of $S$ and $M_{A}$ proved above, one has $x \in \mathbb{N}^{I}$ with $\sum_{j \in I} a_{i j} x_{j}=0$ for $x_{j}=\pi_{j}(s)$ with $j \in I$ and $s \in S$. Since $\pi_{j_{2}}$ is essential, there exists by Lemma 2.3 an $s \in S$ with $\pi_{j_{2}}(s)=0$ and $\pi_{j_{1}}(s)>0$. Therefore, there must be some $j \in I$ with $a_{i j}<0$. Thus, $I_{+} \neq \emptyset$ implies $I_{-} \neq \emptyset$. Similarly, $I_{-} \neq \emptyset$ implies $I_{+} \neq \emptyset$. Therefore, there exist $j_{1} \in I_{+}$and $k_{1} \in I_{-}$. Suppose now $I_{+}=\left\{j_{1}\right\}$. Since $\pi_{j_{1}}$ is essential, by Lemma 2.3 there exists $s \in S$ with $\pi_{j_{1}}(s)=0$ and $\pi_{k_{1}}(s)>0$. But then, for $x=\left(\pi_{j}(s)\right)_{j}$, we obtain $0=-a_{i j_{1}} x_{j_{1}}=\sum_{j \neq j_{1}} a_{i j} x_{j}<0$, which is a contradiction.

Thus we must have that $\left|I_{+}\right| \geq 2$. Similarly, $\left|I_{-}\right| \geq 2$. This proves the statement about $A_{21}$ in Part iii) of Theorem 3.1.

\section{Remarks 3.2.}

1. The Diophantine monoid $M_{A}$ in Theorem 3.1 ii) consists of two different kinds of equations. The first $k$ equations are of type 1 and the remaining $h$ equations are of type 2 in the sense of [2, Theorem 1.3]. The latter type does not occur if and only if $h=0$, or, equivalently, $r=e$ which for $e \leq 3$ must happen by Theorem 3.1 iii).

2. Concerning Krull monoids $S$ with infinitely many essential states, Step I in the proof of Theorem 3.1 can be used provided the module $M=\varphi(Q(S))$ meets the assumptions made there. In general, a submodule $M$ of $\mathbb{Z}^{(I)}$ does not satisfy these assumptions as the following simple example shows. Let $I=\mathbb{N},\left(u_{i}\right)_{i \in \mathbb{N}}$ the standard basis of $\mathbb{Z}^{(\mathbb{N})}, \mathbb{Q}=\left\{q_{i}\right\}_{i \in \mathbb{N}}$ and $\tau: \mathbb{Z}^{(\mathbb{N})} \longrightarrow \mathbb{Q}$ be the $\mathbb{Z}$-homomorphism defined by $\tau\left(u_{i}\right)=q_{i}$ for all $i \in \mathbb{N}$. If the submodule $M=\operatorname{Ker} \tau$ of $\mathbb{Z}^{(\mathbb{N})}$ would satisfy the assumptions of Step I, then by $(2) \mathbb{Q} \simeq \mathbb{Z}^{(\mathbb{N})} / M$ should be isomorphic to a direct sum of copies of $\mathbb{Z}$ and $\mathbb{Z}_{\alpha}$ (which is not the case). If, however, for $I$ infinite $\mathrm{Cl}(S)$ is finitely generated, then one 
can argue that $S$ is isomorphic to a monoid $M_{A}$ where the number of rows of $A$ is rank $\mathrm{Cl}(S)$ and the "number" of columns of $A$ is given by the cardinality of $I$ augmented by a finite set. Similarly, if $\mathrm{Cl}(S)$ is free, then $S$ is isomorphic to a monoid $M_{A}$ with $|I \backslash J|$ equations in $|I|$ unknowns.

From Theorem 3.1 we obtain the following characterization of Diophantine monoids.

Corollary 3.3. For a reduced monoid $S$ the following statements are equivalent:

i) $S$ is a Krull monoid with finitely many essential states.

ii) $S$ is isomorphic to a Diophantine monoid.

iii) $S$ is isomorphic to a monoid $W \cap \mathbb{N}^{n}$ for a vector subspace $W$ of $\mathbb{Q}^{n}$ and some $n \geq 1$.

iv) $S$ is isomorphic to a full and expanded submonoid $T$ of $\mathbb{N}^{n}$ (i.e., $x, y \in$ $T, y-x \in \mathbb{N}^{n}$ imply $y-x \in T$, and $k z \in T$ for $k \geq 1, z \in \mathbb{N}^{n}$, implies $z \in T)$.

v) $S$ is root-closed and finitely generated.

Proof. i) $\Rightarrow$ ii) follows directly from Theorem 3.1. Obviously, ii) $\Rightarrow$ iii). Since $T=W \cap \mathbb{N}^{n}$ is full and expanded, iii) $\Rightarrow$ iv). From iv) it follows by Dickson's Lemma (see [14, Theorem 5.1]) that $T$, and hence $S$, is finitely generated. Since $T$ is expanded, $S$ must be root-closed (i.e., $k z \in S$ for $k \geq 1, z \in Q(S)$ implies $z \in S)$. This yields iv) $\Rightarrow \mathrm{v})$. Finally, v) $\Rightarrow \mathrm{i}$ ) follows from a well-known theorem of Halter-Koch [7, Theorem 5] and the main result in [13], by which a root-closed and finitely generated monoid is a Krull monoid described by finitely many states.

\section{Remarks 3.4.}

1. In [13, Corollary 1] a description of the class group is given by employing terminology and methods from convex analysis. Using results from [13], in [10, Proposition 2] it is shown that a monoid $S$ satisfying $Q(S)=\mathbb{Z}^{n}$ is isomorphic to the monoid of nonnegative solutions of a homogeneous system of integral linear equations if and only if $S$ is a Krull monoid which holds if any only if $S$ is finitely generated and root-closed. The method used in the proof of Theorem 3.1 is direct and does not involve convex analysis. Moreover, Theorem 3.1 describes the representing matrix $A \in \mathbb{Z}^{m \times n}$ in terms of the class group and the number of essential states of $S$. This yields in particular that $m=\operatorname{rank} \mathrm{Cl}(S)$.

2. Corollary 3.3 applies in particular to additive submonoids $S$ of $\mathbb{N}^{n}$. For this case it has been shown in [8, Lemma 3] that $S$ is the set of solutions in the nonnegative integers of a system of homogeneous linear equations with rational coefficients if and only if $S=W \cap \mathbb{N}^{n}$ 
for a vector subspace $W$ of $\mathbb{Q}^{n}$ or, equivalently, $S$ is full and expanded. An additive submonoid $S$ of $\mathbb{N}^{n}$ is called a full affine semigroup if $S=M \cap \mathbb{N}^{n}$ for a subgroup $M$ of $\mathbb{Z}^{n}$ or, equivalently, $S=Q(S) \cap \mathbb{N}^{n}$ (see [14, Chapter 7] for more about these semigroups). Obviously, such a semigroup is root-closed, but it need not be expanded as the example $S=2 \mathbb{N}$ shows. Furthermore, such a semigroup is a Krull monoid with finitely many essential states. From Corollary 3.3 one concludes that an arbitrary reduced monoid is a Krull monoid with finitely many essential states if and only if it is isomorphic to a full affine semigroup.

By Theorem 3.1 Part ii), we know how to describe an arbitrary Krull monoid $S$ having a finite number of essential states by a particular matrix adapted to $S$. The following Lemma addresses, conversely, a Krull monoid given by an arbitrary matrix. For a matrix $A$, let im $A$ denote the image of the mapping induced by $A$.

Lemma 3.5. Let $S \simeq M_{A}$ with $A \in \mathbb{Z}^{m \times n}, m \geq 1, n \geq 1$ and weight $w\left(M_{A}\right)=1$. Arrange $A$ such that the first e canonical projections are exactly the normal essential states of $M_{A}$ and let $A=\left[A^{\prime} A^{\prime \prime}\right]$ where $A^{\prime} \in$ $\mathbb{Z}^{m \times e}, A^{\prime \prime} \in \mathbb{Z}^{m \times(n-e)}$. The following statements hold:

i) $\mathrm{Cl}(S) \simeq \operatorname{im} A^{\prime} /\left(\operatorname{im} A^{\prime} \cap \operatorname{im} A^{\prime \prime}\right)$.

ii) $n=\operatorname{rank} A+\operatorname{rank} Q(S)=\operatorname{rank} A^{\prime \prime}+e$.

iii) $\operatorname{rank} A^{\prime}-\operatorname{rank}\left(\operatorname{im} A^{\prime} \cap \operatorname{im} A^{\prime \prime}\right)=$ free $\operatorname{rank} \mathrm{Cl}(S) \leq \operatorname{rank} \mathrm{Cl}(S) \leq$ rank $A^{\prime}$.

iv) $\operatorname{rank} \mathrm{Cl}(S) \leq m$ and $\operatorname{rank} \mathrm{Cl}(S)+\operatorname{rank} Q(S) \leq n$.

In particular, if $A^{\prime}=A$, then one has $\mathrm{Cl}(S) \simeq \mathbb{Z}^{k}, k=\operatorname{rank} A$ and $\operatorname{rank} \mathrm{Cl}(S)+\operatorname{rank} Q(S)=n$.

Proof. It can be assumed that $S=M_{A}$. By assumption the divisor theory $\varphi: Q(S) \longrightarrow \mathbb{Z}=E A$ is given by $\varphi\left(y_{1}, \ldots, y_{n}\right)=\left(y_{1}, \ldots, y_{e}\right)$. From Lemma 2.6 it follows that $Q(S)=\operatorname{Ker} A$.

i) Consider $f: \operatorname{im} A^{\prime} \longrightarrow \mathrm{Cl}(S)$ defined by $A^{\prime} x \longmapsto x+\varphi(Q(S))$ for $x \in$ $\mathbb{Z}^{e}$. The mapping $f$ is well-defined. For, if $A^{\prime} x=0$, then $y=\left[\begin{array}{l}x \\ 0\end{array}\right] \in$ $Q(S)$ and, hence, $x=\varphi(y) \in \varphi(Q(S))$. Obviously, $f$ is surjective. Furthermore, if $A^{\prime} x \in \operatorname{Ker} f$ then $x \in \varphi(Q(S))$ and $A^{\prime} x+A^{\prime \prime} y=0$ for some $y \in \mathbb{Z}^{n-e}$. This shows $\operatorname{Ker} f \subseteq \operatorname{im} A^{\prime} \cap \operatorname{im} A^{\prime \prime}$. Conversely, if $A^{\prime} x \in \operatorname{im} A^{\prime \prime}$, then $A^{\prime} x+A^{\prime \prime} y=0$ for some $y \in \mathbb{Z}^{n-e}$ and $x=$ $\varphi\left(\left[\begin{array}{l}x \\ y\end{array}\right]\right) \in \varphi(Q(S))$. This shows $\operatorname{im} A^{\prime} \cap \operatorname{im} A^{\prime \prime} \subseteq \operatorname{Ker} f$. Thus, $\operatorname{Ker} f=\operatorname{im} A^{\prime} \cap \operatorname{im} A^{\prime \prime}$ and we have the short exact sequence

$$
0 \longrightarrow \operatorname{im} A^{\prime} \cap \operatorname{im} A^{\prime \prime} \stackrel{i}{\rightarrow} \operatorname{im} A^{\prime} \stackrel{f}{\rightarrow} \mathrm{Cl}(S) \longrightarrow 0,
$$


where $i$ denotes the embedding. Therefore, $\mathrm{Cl}(S) \simeq \operatorname{im} A^{\prime} /\left(\mathrm{im} A^{\prime} \cap\right.$ $\left.\operatorname{im} A^{\prime \prime}\right)$.

ii) Obviously, $n=\operatorname{rankim} A+\operatorname{rank} \operatorname{Ker} A$. Since $\operatorname{Ker} A=Q(S)$ it follows that $n=\operatorname{rank} A+\operatorname{rank} Q(S)$. Also, $n-e=\operatorname{rankim} A^{\prime \prime}+\operatorname{rank} \operatorname{Ker} A^{\prime \prime}$. We show that $\operatorname{Ker} A^{\prime \prime}=0$ which proves ii). If $z \in \operatorname{Ker} A^{\prime \prime}$, then $y,-y \in$ $\operatorname{Ker} A$ for $y=\left[\begin{array}{l}0 \\ z\end{array}\right]$. It follows that $y,-y \in Q\left(M_{A}\right)$ and $\pi_{i}(-y)=$ $\pi_{i}(y) \in \mathbb{N}$ for every $i \in I=\{1,2, \ldots, e\}$. Since $\pi_{i}, i \in I$ are the essential projections of $M_{A}$, we obtain that $y,-y \in M_{A}$. Since $M_{A} \subseteq$ $\mathbb{N}^{n}$ we must have that $y=0$ and, hence, $z=0$.

iii) Follows directly from i).

iv) From iii) we have that

$$
\operatorname{rank} \mathrm{Cl}(S) \leq \operatorname{rank} A^{\prime} \leq \min \{m, \operatorname{rank} A\}
$$

and using ii) we obtain

$$
\operatorname{rank} \mathrm{Cl}(S)+\operatorname{rank} Q(S) \leq \operatorname{rank} A+\operatorname{rank} Q(S)=n .
$$

The result for $A^{\prime}=A$ follows immediately since in this case the proof for i) shows that $\operatorname{Ker} f=\{0\}$.

Concerning the representation of a monoid by a matrix, it is a natural question to ask for a matrix with a minimal number of rows and columns, respectively.

Definition 3.6. For a monoid $S$ the row degree of $S$ is defined by

$$
d_{r}(S)=\min \left\{m \in \mathbb{N} \mid S \simeq M_{A} \text { for } A \in \mathbb{Z}^{m \times n}\right\}
$$

and the column degree of $S$ is defined by

$$
d_{c}(S)=\min \left\{n \in \mathbb{N} \backslash\{0\} \mid S \simeq M_{A} \text { for } A \in \mathbb{Z}^{m \times n}\right\} .
$$

Remark 3.7. Since we defined for $A \in \mathbb{Z}^{m \times n}$ that $M_{A}=\mathbb{N}^{n}$ if $m=0$, it follows that $d_{r}(S)=0$ if and only if $S \simeq \mathbb{N}^{n}$ for some $n$. Also, for $S \simeq \mathbb{N}^{n}$ it follows from Lemma 3.5 ii) that $d_{c}(S)=n$.

From Theorem 3.1 together with Lemma 3.5, we obtain the theorem announced in the Introduction.

Theorem 3.8. For a reduced monoid $S$ which is root-closed and finitely generated the following holds:

i) Row degree and column degree of $S$ are finite and

$$
d_{r}(S)=\operatorname{rank} \mathrm{Cl}(S), d_{c}(S)=\operatorname{rank} \mathrm{Cl}(S)+\operatorname{rank} Q(S) .
$$

ii) There exists a matrix $A \in \mathbb{Z}^{m \times n}$ with $m=d_{r}(S)$ and $n=d_{c}(S)$ such that $S$ is isomorphic to the Diophantine monoid $M_{A}$.

Proof. By Corollary 3.3, $S$ is a Krull monoid with finitely many essential states. 
i) By Theorem 3.1, $d_{r}(S) \leq \operatorname{rank} \mathrm{Cl}(S)<\infty$ and $d_{c}(S) \leq \operatorname{rank} \mathrm{Cl}(S)+$ $\operatorname{rank} Q(S)<\infty$. Suppose $S \simeq M_{A}$ for $A \in \mathbb{Z}^{m \times n}$. We may assume that $w\left(M_{A}\right) \neq 0$ and, by Lemma 2.6 , that $S \simeq M_{A D}$ with $w\left(M_{A D}\right)=1$. From Lemma 3.5 iv) we obtain $\operatorname{rank} \mathrm{Cl}(S) \leq m$ and $\operatorname{rank} \mathrm{Cl}(S)+$ $\operatorname{rank} Q(S) \leq n$. This proves i).

ii) Follows immediately from i) and Theorem 3.1.

\section{Some consequences and examples.}

The results of the previous section can be used to check if a Diophantine monoid is factorial or half-factorial.

Proposition 4.1. Let $S \simeq M_{A}$ and $A=\left[A^{\prime} A^{\prime \prime}\right]$ be given as in Lemma 3.5.

i) $S$ is factorial if and only if each column of $A^{\prime}$ is in the column space of $A^{\prime \prime}$.

ii) If any two columns of $A^{\prime}$ not in the column space of $A^{\prime \prime}$ have their difference in the column space of $A^{\prime \prime}$, then $S$ is half-factorial.

Proof. It is well-known that any Krull monoid $S$ is factorial if and only if $\mathrm{Cl}(S)=\{0\}$ and that $S$ is half-factorial if $\mathrm{Cl}(S) \simeq \mathbb{Z}_{2}$ (cf. [12, Proposition 2]).

i) By Lemma 3.5, $\mathrm{Cl}(S)=\{0\}$ if and only if im $A^{\prime} \subseteq \operatorname{im} A^{\prime \prime}$. Therefore, $S$ is factorial if and only if each column of $A^{\prime}$ is in the $\mathbb{Z}$-span of the columns of $A^{\prime \prime}$.

ii) If all columns of $A^{\prime}$ are in im $A^{\prime \prime}$ then by i) $S$ is factorial and, a fortiori, half-factorial. Suppose there is a column $A_{i_{0}}^{\prime}$ of $A^{\prime}$ which is not in $\operatorname{im} A^{\prime \prime}$. By assumption, for any column $A_{i}^{\prime} \notin \operatorname{im} A^{\prime \prime}$ it holds that $A_{i}^{\prime}-$ $A_{i_{0}}^{\prime} \in \operatorname{im} A^{\prime \prime}$. Therefore, for each column $A_{i}^{\prime}$ of $A^{\prime}$ either $A_{i}^{\prime} \in \operatorname{im} A^{\prime \prime}$ or $A_{i}^{\prime} \in A_{i_{0}}^{\prime}+\operatorname{im} A^{\prime \prime}$. Lemma 3.5 implies that $\mathrm{Cl}(S) \simeq \mathbb{Z}_{2}$ and, hence, $S$ is half-factorial.

Remark 4.2. Neither the condition given in ii) nor the condition $\mathrm{Cl}(S) \simeq$ $\mathbb{Z}_{2}$ are necessary for half-factoriality. This is so even for $m=1$; see [2] for various sufficient or necessary conditions of half-factoriality in this particular case. 
In [2, Theorem 1.3], the current authors found a formula for computing the class group of a Diophantine monoid given by just one equation. The results of Section 3 yield a simpler proof of this formula, as well as an additional characterization of such monoids.

\section{Proposition 4.3.}

i) A monoid $S$ is isomorphic to a nonfactorial Diophantine monoid given by just one equation if and only if $S$ is reduced, root-closed, and finitely generated with rank $\mathrm{Cl}(S)=1$.

ii) Let $S=M_{A}$ with $A=\left[a_{1} a_{2} \ldots a_{n}\right] \in \mathbb{Z}^{1 \times n}$ and, without restriction, $a_{i} \neq 0$ for all $i$, not all $a_{i}$ of equal sign and $\operatorname{gcd}\left(a_{1}, \ldots, a_{n}\right)=1$. There are exactly two possible cases:

a) $\operatorname{Cl}(S) \simeq \mathbb{Z}_{\alpha}$ with $\alpha \in \mathbb{N}$. This case occurs if and only if all $a_{i}$ except one, say $a_{n}$, are of equal sign. Thus we have $\alpha=\frac{\left|a_{n}\right|}{c}$, where $c=\prod_{i=1}^{n-1} c_{i}$ and $0<c_{i}=\operatorname{gcd}\left(\left|a_{j}\right| \mid j \neq i\right)$ for $1 \leq i \leq n$.

b) $\mathrm{Cl}(S) \simeq \mathbb{Z}$. This case occurs if and only if there are at least two $a_{i}$ with positive sign and at least two $a_{i}$ with negative sign.

Proof. The proof of i) follows directly from Theorem 3.8. For ii), let $\widetilde{a_{j}}=$ $\frac{a_{j}}{d_{j}}$ with $d_{j}=\prod_{i \neq j} c_{i}$. For $\widetilde{A}=\left[\widetilde{a_{1}} \ldots \widetilde{a_{n}}\right]$ the mapping $\psi: M_{A} \longrightarrow M_{\widetilde{A}}$, $\psi_{i}\left(x_{1}, \ldots, x_{n}\right)=\frac{x_{i}}{c_{i}}$ is a monoid isomorphism. Hence $\mathrm{Cl}\left(M_{A}\right) \simeq \mathrm{Cl}\left(M_{\widetilde{A}}\right)$. Furthermore, $w\left(M_{\widetilde{A}}\right)=1$. Thus, we can assume that $c_{i}=1$ for all $i, c=1$ and $w\left(M_{A}\right)=1$.

a) Let $a_{i}>0$ for $1 \leq i \leq n-1$ and $a_{n}<0$. Then $A=\left[A^{\prime} A^{\prime \prime}\right]$ with $A^{\prime}=\left[a_{1} \ldots a_{n-1}\right]$ and $A^{\prime \prime}=a_{n}=-\alpha$. Since $c_{n}=1$, we have that $1 \in \operatorname{im} A^{\prime}$ and, hence, im $A^{\prime}=\mathbb{Z}$. Obviously, im $A^{\prime \prime}=\alpha \mathbb{Z}$ and from Lemma 3.5 i) we obtain $\mathrm{Cl}\left(M_{A}\right) \simeq \mathbb{Z}_{\alpha}$.

b) From the assumption in b), all projections must be essential and hence, $A=A^{\prime}$. From $\operatorname{gcd}\left(a_{1}, \ldots, a_{n}\right)=1$ it follows that $\operatorname{im} A^{\prime}=\mathbb{Z}$ and Lemma 3.5 yields $\mathrm{Cl}\left(M_{A}\right) \simeq \mathbb{Z}$.

The following example illustrates the concept of row degree and column degree, respectively and the statements made in Theorem 3.8.

Example 4.4. Consider the Diophantine monoid

$$
S=\left\{x \in \mathbb{N}^{5} \mid x_{1}+x_{2}=x_{4}+x_{5}, x_{2}+x_{5}=x_{3}+x_{4}\right\} .
$$


Obviously, $S=M_{A}$ for $A=\left[\begin{array}{ccccc}1 & 1 & 0 & -1 & -1 \\ 0 & 1 & -1 & -1 & 1\end{array}\right] \in \mathbb{Z}^{2 \times 5}$. All canonical projections are normal and by Lemma 2.3 the projections $\pi_{1}$ to $\pi_{4}$ are essential while $\pi_{5}$ is not. We have $w\left(M_{A}\right)=1$ and $A=\left[A^{\prime} A^{\prime \prime}\right]$ with $A^{\prime}=\left[\begin{array}{rrrr}1 & 1 & 0 & -1 \\ 0 & 1 & -1 & -1\end{array}\right]$ and $A^{\prime \prime}=\left[\begin{array}{r}-1 \\ 1\end{array}\right]$. It follows that $\operatorname{im} A^{\prime}=$ $\mathbb{Z} \oplus \mathbb{Z}$, im $A^{\prime \prime}=\mathbb{Z}\left[\begin{array}{l}-1 \\ +1\end{array}\right]$, and, by Lemma $\left.3.5 \mathrm{i}\right), \mathrm{Cl}\left(M_{A}\right) \simeq \mathbb{Z}$. Since $\operatorname{rank} Q\left(M_{A}\right)=3$, it follows from Theorem 3.8 i) that $d_{r}(S)=1$ and $d_{c}(S)=1+3=4$. By Theorem 3.8 ii) there exists a matrix $B \in \mathbb{Z}^{1 \times 4}$ such that $M_{A}$ is isomorphic to the Diophantine monoid $M_{B}$ given by the smaller matrix $B$. Indeed, one can reduce the given system of two equations to just one equation as follows. Eliminating $x_{5}=x_{1}+x_{2}-x_{4}$ yields $x_{2}+\left(x_{1}+x_{2}-x_{4}\right)=x_{3}+x_{4}$. That is $x_{1}+2 x_{2}-x_{3}-2 x_{4}=0$. One has to make sure, however, that for any solution in $\mathbb{N}$ of the latter equation, it automatically holds that $x_{5}=x_{1}+x_{2}-x_{4} \geq 0$. This is obvious in case of $x_{2} \geq x_{4}$. If $x_{2} \leq x_{4}$, then one obtains $x_{1}+x_{2}-x_{4} \geq x_{1}+2\left(x_{2}-x_{4}\right)=x_{3} \geq 0$.

By Theorem 3.8, one can easily check if a given Diophantine monoid can be described by a smaller matrix without actually carrying out the elimination procedure as in the above example. This latter process might be quite difficult in general.

The following example also illustrates Theorem 3.8, and essentially covers all class group possibilities of rank 2. In contrast to Example 4.4, here there is no possible smaller description of the given monoids.

Example 4.5. Let $G$ be a finitely generated abelian group of rank 2 . We show how to construct a Diophantine monoid $S$ defined by 2 equations such that $\mathrm{Cl}(S) \simeq G$. There are three cases to consider.

(a) Suppose $G \simeq \mathbb{Z}_{n} \oplus \mathbb{Z}_{k n}$ for positive integers $n>1$ and $k \geq 1$. Let

$$
S=\left\{x \in \mathbb{N}^{5} \mid x_{1}+x_{3}=n x_{4}, x_{2}+x_{3}=k n x_{5}\right\} .
$$

Obviously, $S=M_{A}$ for $A=\left[\begin{array}{rrrrr}1 & 0 & 1 & -n & 0 \\ 0 & 1 & 1 & 0 & -k n\end{array}\right] \in \mathbb{Z}^{2 \times 5}$. It is easy to check that all canonical projections are normal and, using Lemma 2.3, that the projections $\pi_{1}, \pi_{2}, \pi_{3}$ are essential while $\pi_{4}, \pi_{5}$ are not. Thus, $w\left(M_{A}\right)=1$ and $A=\left[A^{\prime} A^{\prime \prime}\right]$ with $A^{\prime}=\left[\begin{array}{lll}1 & 0 & 1 \\ 0 & 1 & 1\end{array}\right]$ and $A^{\prime \prime}=\left[\begin{array}{rr}-n & 0 \\ 0 & -k n\end{array}\right]$. It follows 
that $\operatorname{im} A^{\prime}=\mathbb{Z} \oplus \mathbb{Z}$ and $\operatorname{im} A^{\prime \prime}=n \mathbb{Z} \oplus k n \mathbb{Z}$. Therefore, by Lemma 3.5 i), $\mathrm{Cl}(S) \simeq(\mathbb{Z} \oplus \mathbb{Z}) /(n \mathbb{Z} \oplus k n \mathbb{Z}) \simeq \mathbb{Z}_{n} \oplus \mathbb{Z}_{k n}$. Since $\operatorname{rank} Q(S)=3$, by Theorem 3.8 we have that $d_{r}(S)=2$ and $d_{c}(M)=2+3=5$. Therefore, the Diophantine monoid $S$ can neither be described by one equation alone, nor by less than five variables.

(b) Suppose $G \simeq \mathbb{Z} \oplus \mathbb{Z}_{n}$ for some positive integer $n>1$. Let

$$
S=\left\{x \in \mathbb{N}^{6} \mid x_{1}+x_{2}-x_{3}-x_{4}=0, x_{1}+x_{3}+x_{5}=n x_{6}\right\} .
$$

Clearly, $S=M_{A}$ for $A=\left[\begin{array}{rrrrrr}1 & 1 & -1 & -1 & 0 & 0 \\ 1 & 0 & 1 & 0 & 1 & -n\end{array}\right] \in \mathbb{Z}^{2 \times 6}$. As in (a), $w\left(M_{A}\right)=1$, the projections $\pi_{1}, \pi_{2}, \pi_{3}, \pi_{4}$ and $\pi_{5}$ are essential and $\pi_{6}$ is not essential. Hence, if $A^{\prime}=\left[\begin{array}{rrrrr}1 & 1 & -1 & -1 & 0 \\ 1 & 0 & 1 & 0 & 1\end{array}\right]$ and $A^{\prime \prime}=\left[\begin{array}{r}0 \\ -n\end{array}\right]$, then $\operatorname{im} A^{\prime}=\mathbb{Z} \oplus \mathbb{Z}$ and $\operatorname{im} A^{\prime \prime}=n \mathbb{Z}$. Thus, $\operatorname{Cl}(S) \simeq(\mathbb{Z} \oplus \mathbb{Z}) / n \mathbb{Z} \simeq \mathbb{Z} \oplus \mathbb{Z}_{n}$. Since $\operatorname{rank} Q(S)=4$, we have that $d_{r}(S)=2$ and $d_{c}(M)=2+4=6$.

(c) Suppose that $G \simeq \mathbb{Z} \oplus \mathbb{Z}$. Let

$$
S=\left\{x \in \mathbb{N}^{6} \mid x_{1}+x_{3}-x_{4}-x_{6}=0, x_{2}+x_{3}-x_{5}-x_{6}=0\right\} .
$$

Clearly, $S=M_{A}$ for $A=\left[\begin{array}{rrrrrr}1 & 0 & 1 & -1 & 0 & -1 \\ 0 & 1 & 1 & 0 & -1 & -1\end{array}\right] \in \mathbb{Z}^{2 \times 6}$. As above, $w\left(M_{A}\right)=1$, but here all projections are essential. Thus, $A^{\prime}=A$ and Lemma 3.5 yields $\mathrm{Cl}(S) \simeq \mathbb{Z}^{2}$. Since $\operatorname{rank} Q(S)=4$, we have that $d_{r}(S)=2$ and $d_{c}(M)=2+4=6$.

To close Section 4, we present an algorithm which reflects the techniques developed in Sections 2 and 3. The method is based on having the complete set of minimal solutions over the nonnegative integers of the system $A x=0$. An algorithm for that computation, by García-Sánchez and Rosales, can be found in [14, Section 3, p. 80]. Before proceeding, we will require one additional result. Let $M$ be a finitely generated submonoid of $\left(\mathbb{N}^{n},+\right)$ and $u_{1}, \ldots, u_{t}$ the irreducible elements of $M$. Let $V$ and $W$ be the $\mathbb{Q}$-vector spaces generated by $u_{1}, \ldots, u_{t}$ and $u_{2}-u_{1}, \ldots, u_{t}-u_{1}$ respectively. Then $\operatorname{dim} V-\operatorname{dim} W \leq 1$ and $V=W$ if and only if $u_{1} \in W$.

Lemma 4.6. Let $M, V$ and $W$ be as above. The following statements are equivalent:

i) $M$ is half factorial

ii) $\operatorname{dim} V=1+\operatorname{dim} W$.

Proof. For i) $\Rightarrow$ ii), assume that $u_{1}=\sum_{j=2}^{t} \alpha_{j}\left(u_{j}-u_{1}\right) \in W$ with each $\alpha_{j} \in \mathbb{Q}$. Then $k_{1} u_{1}=\sum_{j=2}^{t} k_{j}\left(u_{j}-u_{1}\right)$ for suitable $k_{1}, \ldots, k_{t} \in \mathbb{Z}, k_{1} \neq 0$. Since $\left(k_{1}+\sum_{j=2}^{t} k_{j}\right) u_{1}-\sum_{j=2}^{t} k_{j} u_{j}=0$, the monoid $M$ is not half factorial. 
For ii) $\Rightarrow$ i), let $r_{1}, \ldots, r_{t} \in \mathbb{Z}$ with

$$
\sum_{j=1}^{t} r_{j} u_{j}=0=\sum_{j=2}^{t} r_{j}\left(u_{j}-u_{1}\right)+\left(\sum_{j=1}^{t} r_{j}\right) u_{1} .
$$

Since $u_{1} \notin W$ it follows that $\sum_{j=1}^{t} r_{j}=0$.

Algorithm 4.7. Assume that $A \in \mathbb{Z}^{m \times n}$. The following algorithm calculates the class group of $M_{A}$ and determines whether or not $M_{A}$ is halffactorial. Suppose that $\left(u_{\tau} \mid 1 \leq \tau \leq t\right) \neq \emptyset$ is the family of all irreducible elements of the monoid $M_{A}$. Let $C:=\left(u_{i \tau}\right) \in \mathbb{N}^{n \times t}$ be the matrix with column vectors $u_{\tau}$ and row vectors $v_{i}:=\left(u_{i \tau}\right) \in \mathbb{N}^{1 \times t}, 1 \leq i \leq n$. Let $C_{1}:=\left(u_{i \tau}-u_{i 1}\right) \in \mathbb{Z}^{n \times t}$. By Lemma 4.6, $M_{A}$ is not half factorial if and only if $\operatorname{im}_{\mathbb{Z}} C=\operatorname{im}_{\mathbb{Z}} C_{1}$. The calculation of the class group proceeds as follows.

I) Reduction of the system. Let $v_{i}:=\left(u_{i \tau}\right) \in \mathbb{N}^{1 \times t}, 1 \leq i \leq n$, be the $i$-th row vector of the matrix $C$.

Step 1): For all $i \in\{1, \ldots, n\}$, if $v_{i}=0$, then cancel the $i$-th row of $C$.

Step 2): For all $i, j \in\{1, \ldots, n\}$ with $i<j$, if $\lambda v_{i}=v_{j}$ for some $\lambda \in \mathbb{Q}$, then cancel the $j$-th row of $C$.

After these canceling steps, we denote the new matrix again with $C$. Then $C \in \mathbb{Z}^{\widetilde{n} \times t}$ for some $\widetilde{n} \leq n$.

Step 3): For all $j \in\{1, \ldots, \widetilde{n}\}$, calculate $c_{j}:=\operatorname{gcd}\left(u_{j \tau} \mid 1 \leq \tau \leq t\right) \in \mathbb{N}$ and replace the row vector $v_{j}$ of $C$ by $\frac{1}{c_{j}} v_{j}$.

\section{II) Determining the essential states.}

Step 4): For every $i \in\{1, \ldots, \widetilde{n}\}$, define $J_{i}:=\left\{\tau \in\{1, \ldots, t\} \mid u_{i \tau}=0\right\}$. If $J_{i} \neq \emptyset$, calculate the sums

$$
v_{j}^{(i)}:=\sum_{\tau \in J_{i}} u_{j \tau}
$$

for all $j \in\{1, \ldots, \widetilde{n}\}, j \neq i$. Define

$$
I:=\left\{i \in\{1, \ldots, \widetilde{n}\} \mid J_{i} \neq \emptyset, v_{j}^{(i)} \neq 0 \text { for all } j \in\{1, \ldots, \widetilde{n}\}, j \neq i\right\}
$$

and $e:=|I|$. The projections $\pi_{i}, i \in I$, are exactly the essential states of $M$.

III) The final calculation.

Step 5): Let $\widetilde{C} \in \mathbb{N}^{e \times t}$ be the matrix with the row vectors $v_{i}, i \in I$. Transform $\widetilde{C}$ into its Smith normal form, using e.g., the algorithm given in [14]. 
From the Smith normal form one gets $k:=\operatorname{rank} \widetilde{C}$ and $r \geq 0$ elementary divisors $\alpha_{i} \geq 2$ of $\widetilde{C}$ with $\alpha_{i+1} \mid \alpha_{i}$ for $1 \leq i \leq r-1$ if $r \geq 2$. Then

$$
\mathrm{Cl}(M)=\mathbb{Z}^{e-k} \oplus \mathbb{Z} / \alpha_{1} \mathbb{Z} \oplus \cdots \oplus \mathbb{Z} / \alpha_{r} \mathbb{Z} .
$$

Acknowledgment. The authors would like to acknowledge many useful suggestions made by an anonymous referee on an earlier draft of the paper.

\section{References}

[1] S. Chapman and A. Geroldinger, Krull domains and monoids, their sets of lengths, and associated combinatorial problems, Lecture Notes in Pure and Applied Mathematics, Marcel Dekker, 189 (1997), 73-112, MR 98g:13017, Zbl 0897.13001.

[2] S. Chapman, U. Krause and E. Oeljeklaus, Monoids determined by a homogeneous linear Diophantine equation and the half-factorial property, J. Pure Appl. Algebra, 151 (2000), 107-133, MR 2001g:11034.

[3] E. Contejean and H. Devie, An efficient incremental algorithm for solving systems of linear Diophantine equations, Inform. and Comput., 113 (1994), 143-172, MR 96a:11151, Zbl 0809.11015.

[4] E.B. Elliott, On linear homogenous Diophantine equations, Quart. J. Pure Appl. Math., 34 (1903), 348-377.

[5] J.H. Grace and A. Young, The Algebra of Invariants, Cambridge University Press, Cambridge, 1903.

[6] F. Halter-Koch, Halbgruppen mit divisorentheorie, Exposition Math., 8 (1990), 27-66, MR 91c:20091, Zbl 0698.20054.

[7] lem in higher dimensions, in 'Semigroups: Algebraic Theory and Applications to Formal Languages and Codes' (eds. C. Bonzini et al.), World Scientific 1993, 86-93, CMP 1647 172, Zbl 0818.20079.

[8] M. Hochster, Ring of invariants of tori, Cohen-Macauley rings generated by monomials, and polytopes, Ann. of Math., II Ser., 96 (1972), 318-337, MR 46 \#3511, Zbl 0237.14019.

[9] N. Jacobson, The Theory of Rings, Mathematical Surveys II, AMS, Providence, 1943, MR 5,31f, Zbl 0060.07302.

[10] F. Kainrath and G. Lettl, Geometric notes on monoids, Semigroup Forum, 61 (2000), 298-302, CMP 1832 189, Zbl 0964.20037.

[11] U. Krause, On monoids of finite real character, Proc. Amer. Math. Soc., 105 (1989), 546-554, MR 89i:20102, Zbl 0692.20058.

[12] U. Krause and C. Zahlten, Arithmetic in Krull monoids and the cross number of divisor class groups, Mitt. Math. Ges. Hamburg, 12 (1991), 681-696, MR 93b:11142, Zbl 0756.20010.

[13] G. Lettl, Subsemigroups of finitely generated groups with divisor-theory, Monatsh. Math., 106 (1988), 205-210, MR 89m:20078, Zbl 0671.20059.

[14] J.C. Rosales and P.A. García-Sánchez, Finitely Generated Commutative Monoids, Nova Scientific Publishers, 1999, MR 2000d:20074, Zbl 0966.20028. 
[15] R. Stanley, Linear Diophantine equations and local cohomology, Inv. Math., 68 (1982), 175-193, MR 83m:10017, Zbl 0516.10009.

[16] _ Combinatorics and Commutative Algebra, Birkhäuser, Boston, 1983, MR 85b:05002, Zbl 0537.13009.

Received September 25, 2000 and revised November 9, 2001. Part of this work was completed while the first author was on an Academic Leave granted by the Trinity University Faculty Development Committee. Part of this work was completed while the second author was visiting Trinity University during the fall of 1999 as the Eva and O.R. Mitchell Visiting Distinguished Professor of Mathematics.

\section{TRINITY UNIVERSITY}

Department of Mathematics

715 Stadium Drive

San Antonio, Texas 78212-7200

E-mail address: schapman@trinity.edu

UNIVERSITÄT BREMEN

FACHBERICh MATHEMATIK/INFormatik

DW-2800 Bremen, Germany

E-mail address: krause@math.uni-bremen.de

UNIVERSITÄT BREMEN

FACHBERICH MATHEMATIK/INFORMATIK

DW-2800 Bremen, Germany

E-mail address: oel@math.uni-bremen.de 